\section{Isolated Pontine Tuberculoma Presenting as Horizontal Gaze Palsy}

A 14-year-old boy presented with inability to move his eyes to the right since one day. There was no history of fever, headache, vomiting or impairment of vision. Vitals were stable while neurological examination revealed right horizontal gaze palsy with contralateral deviation of eyes (Fig. 1a). There was no diplopia, while nystagmus and Doll's eye movement were absent. Pupillary reflex and fundus examination were normal in both eyes. Respiratory system examination revealed pleural effusion on the left side. A clinical impression of right conjugate gaze palsy with left sided pleural effusion, with the etiological possibility of tuberculosis was kept. Investigations revealed hemoglobin $10 \mathrm{~g} / \mathrm{dL}$, total leucocyte count in normal range, and erythrocyte sedimentation rate of 37 $\mathrm{mm} / \mathrm{h}$. Chest $X$-ray showed left sided pleural effusion and pleural tap revealed straw coloured fluid, with an Adenosine deanimase of $87.5 \mathrm{U} / \mathrm{L}$. Mantoux test was non-reactive. Computerized tomography of head showed a ring enhancing lesion, $8.7 \times 7.9 \mathrm{~mm}$ in the posteromedial aspect of the right side of pons with perilesional edema (Fig. 1b).

The child was started on antitubercular treatment, four drugs with prednisolone at $2 \mathrm{mg} / \mathrm{kg} / \mathrm{day}$. The gaze improved and was completely normal after 4 weeks while the pleural effusion resolved after 6 weeks. However, the child returned 3 weeks later with acute onset of right sided facial nerve palsy, lower motor neuron type (Fig. 1c). Compliance with ATT was good and he was on tapering dose of prednisolone. Magnetic resonance imaging of the brain showed a ring enhancing lesion of size $6.6 \times$ $5.4 \mathrm{~mm}$ in the pons in the same area, with perilesional edema. Prednisolone was continued at $2 \mathrm{mg} / \mathrm{kg} / \mathrm{day}$ for 2 weeks and tapered-off over the next 4 weeks. The facial palsy was completely normal 4 weeks later.

Isolated brainstem tuberculomas are rare, accounting for $2.5-8 \%$ of all intracranial tuberculomas [1]. They commonly present as cranial nerve palsies or focal neurological signs but presentation as gaze palsy without diplopia is rare. [2]. Unilateral gaze palsy can result from either a parapontine reticular formation (PPRF) or a VI nucleus lesion [3]. The distinction between the two can be made by Doll's eye

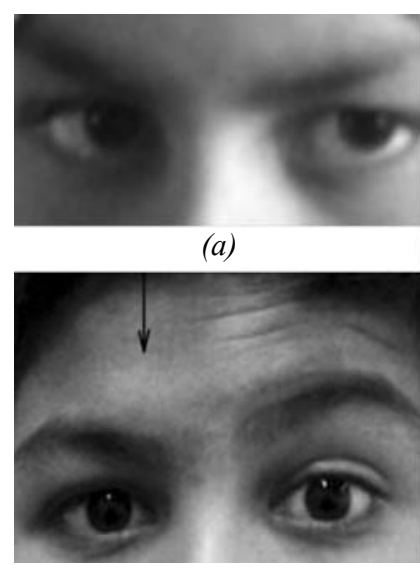

(c)

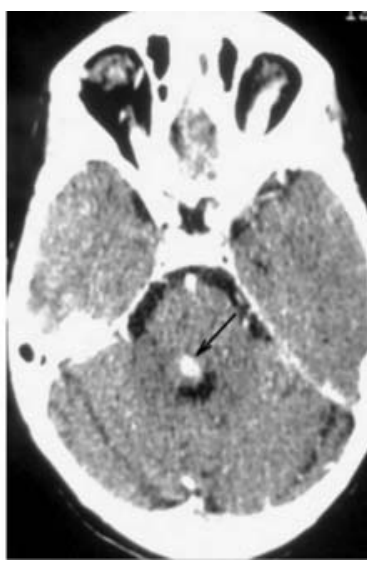

(b)
Fig. 1 (a) Right horizontal gaze palsy with contralateral gaze; (b) Computed tomograpgh of brain showing lesion in pons (black arrow); (c) Normal gaze at 7 weeks with right LMN facial nerve palsy.

manoeuvre, which is preserved in PPRF lesion whereas in a nuclear lesion it is absent [3]. The close proximity of the VII nerve fascicle to the VI nerve nucleus may result in facial nerve palsy, which in our case developed as a paradoxical response seven weeks after starting ATT. Paradoxical response during antituberculosis therapy occurs in about $10-15 \%$ of patients and may develop between 14 to 270 days in HIV negative patients [4]. Brainstem tuberculoma should be considered in the differential diagnosis of neurophthalmologic syndromes, especially in endemic regions.

Acknowledgement: Dr Sanjeev Chaudhary.

*Piyush Gautam and Nivedita Sharma

Department of Pediatrics,

Dr R. P. Medical College and Hospital, Tanda, Kangra,

Himachal Pradesh, India.

*piyushgtm@yahoo.com

\section{REFERENCES}

1. Kumar R, Jain R, Kaur A, Chhabra DK. Brain stem tuberculosis in children. Br J Neurosurg. 2000;14:35661.

2. Lolly P, Rachita S, Satyasundar M. Ophthalmic manifestations of central Nervous system tuberculosisTwo case reports. Indian J Tuberc. 2011;58:196-8.

3. Bronstein AM, Rudge P, Gresty MA, Boulay GDu, Morris J. Abnormalities of horizontal gaze. Clinical, oculographic and magnetic resonance imaging findings. II Gaze palsy and internuclear ophthalmoplegia. J Neurol Neurosurg Psychiatry. 1990;53:200-7.

4. Cheng VC. Paradoxical response during Anti tuberculosis therapy. The Hong Kong Medical Diary. 2006; 2:20-1. 